\title{
Improving Antenatal Asthma Management: A Complex Journey
}

Worsening of asthma during pregnancy is of major concern due to the potential impacts on the mother and fetus. Therefore, the Editor's Pick for this issue is the article is by Chen et al., which delineates the current pharmacological and non-pharmacological interventions that can be utilised in patients with uncontrolled asthma during pregnancy.

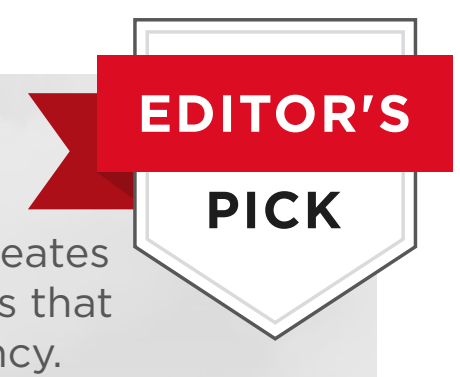
Authors:
Clarissa Chen, ${ }^{1}$ Meng-Wong Taing, ${ }^{1}$ Lucy Burr, ${ }^{2}$ Helen L. Barrett, ${ }^{2}{ }^{*}$ Vicki
L. Clifton ${ }^{2}$
1. School of Pharmacy, The University of Queensland, Brisbane, Australia
2. Mater Research Institute, The University of Queensland, Brisbane, Australia
*Correspondence to vicki.clifton@mater.uq.edu.au

Disclosure:

The authors have declared no conflicts of interest.

Acknowledgements: All authors participated in designing the work and revising and approving the final version.

Received:

01.06 .20

Accepted:

24.08 .20

Keywords:

Evidence-based strategies, coronavirus disease (COVID-19), maternal asthma management, pregnancy.

Citation:

EMJ Respir. 2020;8[1]:97-107.

\begin{abstract}
Asthma is a highly prevalent comorbidity during pregnancy, which can worsen as gestation progresses and is associated with several adverse perinatal outcomes. The adverse outcomes associated with maternal asthma are preventable with appropriate asthma management in pregnancy. However, the prevalence of adverse outcomes has not changed significantly over the last 20 years, even though knowledge and treatments for managing the disease in pregnancy has improved significantly. This is of concern now in the current climate with the coronavirus disease (COVID-19) pandemic and its potential impact on pregnant individuals with asthma. This article will discuss the treatments available for the management of asthma in pregnancy, the barriers for the translation of current knowledge into obstetric practice, and the importance of asthma education and self-management skills.
\end{abstract}

\section{INTRODUCTION}

Worldwide, asthma is one of the most prevalent chronic conditions to potentially have adverse effects in pregnancy and subsequent perinatal outcomes. It affects up to $16 \%$ of females of

reproductive age, resulting in asthma being one of the most common morbidities to affect pregnancy. ${ }^{1}$ Limited progress has been made towards optimising maternal asthma management, particularly in relation to improving adverse outcomes associated with uncontrolled 
asthma during pregnancy. With rapid global impactssuchasthecoronavirus disease(COVID-19) pandemic, this emphasises the need to reassess current strategies and evidence to facilitate more productive approaches. Therefore, this commentary serves to highlight the consequences of uncontrolled asthma during pregnancy and provide insight into existing implications impeding effective management. Additionally, it also aims to evaluate the current literature to understand knowledge gaps and promote future areas of research towards enhancing asthma management during pregnancy.

\section{CONSEQUENCES OF UNCONTROLLED MATERNAL ASTHMA}

Pregnant individuals with asthma often experience unpredictable changes in their asthma symptoms, with 50\% experiencing a loss of asthma control and 20\% experiencing moderate to severe exacerbations. ${ }^{2}$ The reasons for these changes are largely unknown, but can be attributed to the complexity and heterogeneity of the disease itself. 3,4 Patients with asthma are likely to undergo mechanical, immunological, hormonal, and metabolic changes during pregnancy, ultimately experiencing varying degrees of dyspnoea and changes in pulmonary function. 2,4-8 However, the evidence quantifying the extent and significance of such influences are frequently inconsistent and inconclusive., ${ }^{2,4}$ More importantly, uncontrolled maternal asthma and subsequent exacerbations are significant risk factors for adverse maternal and perinatal outcomes. $^{9}$ This includes an increased risk for pre-eclampsia, gestational hypertension, gestational diabetes, caesarean, preterm birth, neonatal hypoxia, and perinatal mortality. ${ }^{9-12}$

New findings have also acknowledged that adverse effects related to uncontrolled maternal asthma extend past birth. A study conducted by Abdullah et al. ${ }^{12}$ was the first to longitudinally investigate the long-term intergenerational impact of maternal asthma exacerbations. Children younger than 5 years of age had a $23 \%$ increased risk of developing early childhood asthma and a $12 \%$ higher chance of developing pneumonia if their mother had experienced asthma exacerbations during their gestation. These findings support the outcomes of smaller crosssectional studies, ${ }^{13,14}$ which identified childhood bronchiolitis and allergic rhinitis as a potential long-term development risk. Additionally, a nationwide Swedish cohort study ${ }^{15}$ found that the risk of developing childhood allergic rhinitis was higher in children born preterm or via caesarean delivery, which are known adverse outcomes of uncontrolled maternal asthma. ${ }^{9}$ These findings suggest that maternal asthma in pregnancy may confer an inherited genetic risk for childhood asthma and allergies, while its lack of control may confer an epigenetic risk for both the fetus and the child. ${ }^{12,15}$ Given these alarming outcomes, there is a need to re-evaluate and optimise evidencebased asthma management strategies during pregnancy to reduce adverse perinatal outcomes and early childhood diseases.

\section{CURRENT ASTHMA MANAGEMENT STRATEGIES}

According to international and national guidelines, 16,17 current maternal asthma therapy is the same as for non-pregnant people with asthma, with goals to optimise symptom control, preserve respiratory function, and provide medication with minimal adverse side effects. Another objective in pregnancy is to prevent maternal hypoxic episodes to maintain fetal oxygenation. 16,17 This is achieved through vigilant monitoring and clinical symptom assessment, self-management education that includes reviewing inhaler technique, medication adherence, and symptom management and application of pharmacotherapies advised by health professionals via a multidisciplinary approach. ${ }^{16,17}$ Counselling for smoking cessation is also recommended as part of asthma education and management because it is a common problem in pregnant females with asthma ${ }^{18,19}$ that contributes to severe exacerbations during pregnancy $^{20}$ and preterm delivery. ${ }^{19}$ Clinical assessment of symptoms, including wheeze, shortness of breath, cough, and chest tightness, are typically measured using spirometry. ${ }^{16,17}$ Guidelines also recommend a stepwise approach for pharmacological intervention, adjusted based on clinical assessment. ${ }^{16,17}$ This primarily includes intermittent use of short-acting $\beta$-agonists as reliever medication with an appropriate dose of inhaled corticosteroids (ICS), combined with or without a long-acting $\beta$-agonist or other alternative treatments. ${ }^{16,17}$ Active management 
during pregnancy is also recommended, with regular monthly reviews, supply of a written asthma plan, and additional management of comorbid conditions. ${ }^{16,17}$ Asthma exacerbations are serious events in pregnancy due to the risk of a hypoxic insult to the fetus and have been recommended to be managed aggressively with oral glucocorticoids as soon as possible.16,17

Recommendations for asthma management during labour and delivery advise asthma medications should be continued during labour. ${ }^{21}$ Asthma symptoms can occur in 10\% of females during labour ${ }^{22}$ and intravenous hydrocortisone during labour for those with severe asthma is recommended to avoid the risk of an exacerbation during labour and/or adrenal insufficiency. Most patients (73\%) return to prepregnancy asthma symptoms within 3 months postpartum and can be titrated back to prepregnancy asthma medications. ${ }^{22}$ Overall, there is a need for improved guidelines for asthma management during pregnancy, birth, and the postpartum period, and this matter has been raised in several recent publications. ${ }^{23,24}$

\section{LIFESTYLE MODIFICATION AND RISK FACTOR REDUCTION}

There are a number of lifestyle factors and comorbidities that are known to affect asthma control and increase exacerbation risk in pregnancy, including smoking, dietary behaviour, rhinitis, gastric reflux, and mental health illnesses or disorders that could be modified with appropriate interventions to reduce the risk of a loss of asthma control or exacerbations during pregnancy. Cigarette use by pregnant individuals with asthma is a major health concern, affecting up to $30 \%$ of females. ${ }^{18}$ From the authors' prospective cohort studies of pregnant individuals with asthma, $29 \%$ of smokers ceased smoking during early pregnancy through usual antenatal care support mechanisms..$^{25}$ In contrast, among those who received additional support through a nurse-led antenatal asthma management programme, 54\% stopped smoking in early pregnancy, suggesting that lifestyle factors can be modified with an appropriate intervention. ${ }^{25}$

Another potentially modifiable behaviour in pregnancy is nutritional intake. In a socially disadvantaged population of pregnant females with asthma consuming a diet high in fat, sugar, and takeaway meals, there was an increased likelihood of uncontrolled asthma during pregnancy, whereas a diet high in protein, fruit, or vegetables was associated with controlled asthma. ${ }^{26}$ Nutritional advice should be considered for individuals with asthma who are obese as there was an increased prevalence of asthma exacerbations among pregnant females who were overweight (51\%) or obese (48\%) compared with those with a healthy weight (25\%). ${ }^{8}$ However, pregnancy-related weight gain appeared to have no impact on exacerbation risk. ${ }^{8}$ These findings provide important evidence that lifestyle modification is a consideration for the antenatal management of maternal asthma, but it remains to be determined whether they represent a key determinant for asthma control in pregnancy.

Comorbidities known to exacerbate asthma, including rhinitis, gastric reflux, and mental health illnesses and disorders, could be managed clinically with maternal antenatal asthma management. Rhinitis is the most common comorbidity, occurring in $65 \%$ of females with asthma during pregnancy, with $20 \%$ of females experiencing pregnancy rhinitis. ${ }^{27}$ The presence of rhinitis was shown to be associated with poorer asthma control and lung function. ${ }^{27} \mathrm{~A}$ history of depression or anxiety in patients with asthma during pregnancy increased therisk ofuncontrolled asthma during pregnancy. ${ }^{28-30}$ Furthermore, anxiety was an independent risk factor for poor asthma control and exacerbations. ${ }^{29,30} \mathrm{~A}$ number of studies have also reported that gastric reflux exacerbates asthma in those who are not pregnant. ${ }^{31}$ A prospective study reported that $80 \%$ of pregnant indiviuals experience some gastric reflux during pregnancy, with it being more severe in those with asthma; ${ }^{32}$ therefore, it is possible asthma control in pregnancy may be influenced by the presence or absence of gastric reflux. These studies highlighted that lifestyle factors and comorbidities could be examined holistically with antenatal asthma management to develop preventive strategies for asthma control in pregnancy and ultimately improve pregnancy-related outcomes. 


\section{EFFECTIVENESS OF \\ PHARMACOLOGICAL AND NON- PHARMACOLOGICAL INTERVENTIONS}

\section{Pharmacological Treatment}

Pharmacological therapy is the current mainstay treatment for effective asthma control in pregnancy. ${ }^{10,33,34}$ Uncontrolled maternal asthma poses a greater risk to the health of the mother and her baby than medication use. 33,34 Table 1 summarises asthma medication safety data with respect to reported doses and adverse events. ${ }^{7,35-38}$

The safety of ICS has been extensively investigated in pregnancy and there is growing evidence supporting ICS/long-acting $\beta$-agonists combinations. ${ }^{10,33,34}$ This strengthens the argument and recommendation, by guidelines, that females entering pregnancy should continue medication/s they were taking prior to conception, if the treatment remains clinically appropriate. Other commonly used asthma medications with pregnancy safety data include systemic steroids, leukotriene receptor antagonists, omalizumab, and, rarely, theophylline..$^{10,33,34}$

The cumulation of pharmacological safety data in pregnancy is progressive; however, may be limited by studies that have small sample sizes, insufficient control for cofounders, and missing data. ${ }^{39}$ Thus, more research is required to improve evidence-based safety data of asthma pharmacological treatment during pregnancy. ${ }^{39}$ Furthermore, asthma pharmacotherapy adjusted in response to lung function and symptom assessment does not always reflect airway inflammation, the target of ICS treatment. ${ }^{40}$ This suggests that decisions in therapy based on clinical assessment alone may be inappropriate, with growing awareness of the heterogeneity of asthma and its differing phenotypes. ${ }^{40}$ Therefore, the correct identification of phenotypic asthma unique to the individual proposes the potential for individualising asthma treatment. ${ }^{41}$ Additionally, as mentioned before, the perceptions concerning the relative safety of medication use in pregnancy is a continuous impediment in optimising maternal asthma management. ${ }^{34}$

\section{Fraction of Exhaled Nitric Oxide-Guided Diagnosis and Asthma Management}

The pursuit for novel, individualising-type treatments is unsurprising, particularly as biomarker-guided therapies, such as the use of the noninvasive measure of fraction of exhaled nitric oxide (FeNO), have already shown to improve asthma control in those who are not pregnant. ${ }^{42,43}$ In particular, as FeNO can be used to identify eosinophilic airway inflammation, it helps guide ICS dose titrations, thereby limiting ICS exposure and exacerbation rates. ${ }^{42,43}$ In pregnancy, the efficacy of FeNO-based therapy has been established in one randomised controlled trial (RCT) study conducted by Powell et al. ${ }^{44}$ The study involved 220 nonsmoking females and reported that one-half of the exacerbations were reduced in the FeNO participants in comparison to the control group. It also reported that there was increased retention of asthma control in the FeNO participants, thereby allowing progressive ICS dose reductions, as well as minimised oral corticosteroid and short-acting $\beta$-agonist use.

Long-term benefits of FeNO treatment in pregnancy have also been investigated, with reports of reduced wheeze and childhood asthma prevalence in children of mothers who received FeNO treatment during pregnancy. ${ }^{45,46}$ Additionally, a qualitative study by Mclaughlin et al. $^{42}$ determined that clinicians found FeNO-guided treatment implementation to be feasible within hospital-based antenatal care settings if resources and education are appropriately provided.

Despite these positive outcomes, limitations have been identified in this approach by Grzeskowiak and Clifton, ${ }^{43}$ including its uncertain applicability in primary clinical care, accessibility, and costeffectiveness. However, continuing studies such as The Breathing for Life Trial ${ }^{40}$ will not only address these limitations to better comprehend the feasibility of FeNO-guided antenatal therapy, but will also include smokers within the pregnant females with asthma cohort. This allows the generalisability of this approach to be better understood, particularly because there is a high proportion of pregnant individuals with asthma who smoke (>20\%). ${ }^{19,43}$ 
Table 1: Summary of the efficacy of asthma medications used in observational trials to maintain or strengthen asthma control in women with asthma.,

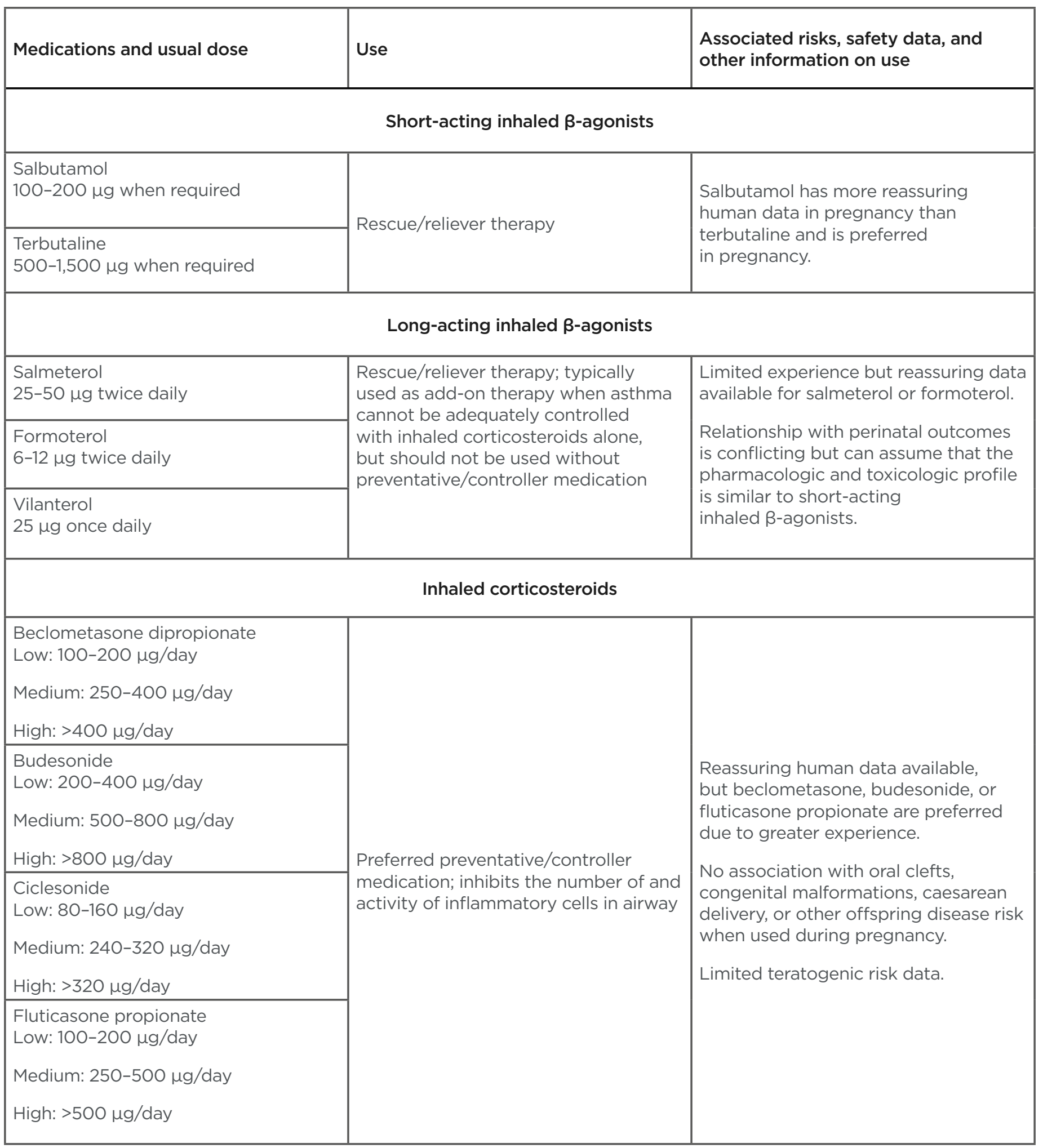


Table 1 continued.

\begin{tabular}{|l|l|l|}
\hline Medications and usual dose & Use & $\begin{array}{l}\text { Associated risks, safety data, and } \\
\text { other information on use }\end{array}$ \\
\hline
\end{tabular}

\section{Systemic corticosteroids}

\begin{tabular}{|l|l|l}
\hline & & $\begin{array}{l}\text { Use of systemic corticosteroids } \\
\text { associated with increased risk of oral } \\
\text { cleft, especially if used in first trimester } \\
\text { of pregnancy. }\end{array}$ \\
$\begin{array}{l}\text { Exacerbation: 40-50 mg once daily for } \\
\text { 5-10 days }\end{array}$ & $\begin{array}{l}\text { Preventative medication and mainstay } \\
\text { treatment of asthma exacerbations; } \\
\text { used if asthma cannot be controlled } \\
\text { maintenance: Variable dose according } \\
\text { to response }\end{array}$ & $\begin{array}{l}\text { Also associated with increased risk } \\
\text { of adverse pregnancy outcomes } \\
\text { (e.g., pre-eclampsia, preterm birth, } \\
\text { small-for-gestational age), but likely } \\
\text { confounded by maternal asthma } \\
\text { severity. }\end{array}$ \\
\hline
\end{tabular}

Leukotriene receptor antagonist

Montelukast

$10 \mathrm{mg}$ once daily

Zafirlukast

$20 \mathrm{mg}$ twice daily
Limited experience.

Reassuring human data available as no risk for major congenital malformations has yet been found.

\section{Other treatments}

\section{Omalizumab}

Subcutaneous 75-375 mg every 2-4 weeks; dose according to weight and serum total IgE level
Anti-lgE monoclonal antibody; addon therapy or inhaled corticosteroid alternative for allergic asthma
Theophylline

300-600 mg/day; dose according to theophylline level or use ideal weight to calculate dose for obese patients
Alternative therapy or alternative adjunctive long-acting bronchodilator therapy; rarely used in pregnancy.
Limited experience but reassuring human data.

No significant increased risk of major congenital malformations, smallfor-gestational age, or prematurity was found for women taking it 8 weeks prior to conception or during pregnancy compared to pregnant asthmatics not taking it.

Carries risk of anaphylaxis, so should not be started during pregnancy.

Reassuring human data available but limited role in practice due to monitoring requirements and associated risk of toxicity (need to ensure serum levels remain between 5 and $12 \mu \mathrm{g} / \mathrm{mL}$ ).

Serum concentration can be increased with alcohol and concurrent use of formoterol. 
It is also important to understand that FeNO cannot be applied as a stand-alone approach, nor does it replace the need for appropriate asthma self-management education. ${ }^{42}$

\section{Asthma Self-Management Education: Optimal Models of Care}

Antenatal asthma self-management education plays a key role in improving maternal asthma control and asthma-related outcomes in pregnancy. Many studies have established the benefits of asthma self-management education. A review by Williamson et al. ${ }^{47}$ reported antenatal asthma self-management education implemented via two interventions, one nurse-led and the other pharmacist-led, showed significant improvements in asthma selfmanagement and control. Patients had improved medication knowledge and inhaler technique, with more than $80 \%$ of the cohort retaining the skills they learnt for at least 6 months postpartum. This indicates that implementation of asthma self-management education could potentially reduce the perceived medicationrelated risks mothers might have.

The RCT conducted by Lim et al. ${ }^{48}$ showed asthma self-management involving pharmacistguided regular monitoring and education could improve maternal asthma outcomes. The study monitored asthma control in 60 pregnant females with asthma at less than 20 weeks' gestation via an Asthma Control Questionnaire (ACQ), where scores $<1.5$ indicated adequately controlled asthma. Patients who received guidance from the multidisciplinary intervention had significant reductions in their ACQ score, with adequately controlled asthma after 6 months compared to $69 \%$ of patients who received usual care. As this RCT was conducted at antenatal clinics in major maternity hospitals, this study also showed that the multidisciplinary intervention could easily be integrated into antenatal settings.

A recent RCT conducted by Zairina et al. ${ }^{49}$ assessed the efficacy of a telehealth-based intervention in providing 72 pregnant patients asthma self-management education. The intervention group were supported by a smartphone application for advice on managing deteriorating asthma symptoms and allowed symptom monitoring via a handheld respiratory device. The study reported that they had superior asthma control and asthma-related quality of life at 6 months post intervention compared to usual care. This unique method alleviated the need to see health professionals in person, while also streamlined the process of communication between patient and health professionals as data were provided electronically. Across all these studies, appropriate implementation of asthma education, particularly via a multidisciplinary approach, had positive influences in improving maternal asthma control. However, more studies are required to see if these interventions actually lead to improved perinatal outcomes..$^{50}$

\section{OTHER LIMITATIONS AND RECOMMENDATIONS FOR IMPROVEMENT}

A lack of uniformity and gaps for specific treatment recommendations exist within current maternal asthma guidelines. A systematic appraisal by McLaughlin et al. ${ }^{23}$ identified that only respiratory guidelines included recommendations for managing maternal asthma, but antenatal guidelines did not. The specific recommendations are also unclear in certain categories; for instance, the need for regular asthma review in pregnancy is not well addressed in guidelines, but should be essential given the unforeseen physiological changes that can occur during pregnancy. ${ }^{23}$ This also raises the concern that there are limited data available to determine which periods of pregnancy are critical for improving maternal asthma control to minimise the probability of an adverse outcome. Moreover, while guidelines have become increasingly more accessible to health professionals, the prevalence of poor outcomes for mothers with asthma and their neonates remain unchanged, indicating that there has been a lack of implementation of these guidelines. ${ }^{31}$ Additionally, a review by Grzeskowiak et al. $^{10}$ found the evaluation of asthma exacerbations are not well defined, particularly as it often relies on the patient's own self-awareness and understanding of symptoms. This is unreliable, particularly as asthma exacerbations by self-help seeking behaviours generally leads to unnecessary emergency department presentations or unscheduled doctor visits. ${ }^{10}$ These limitations and current gaps in knowledge in maternal asthma guidelines are future areas of research to improve current guidelines and limit short- and long-term risks of maternal asthma on mother and fetus. 


\section{BARRIERS PREVENTING EFFECTIVE MANAGEMENT}

A discordance exists between current antenatal asthma management guidelines and clinical practice, which restricts health advances in optimising asthma management in pregnant females. ${ }^{26}$ A contributing factor is that maternal asthma management appears to be reactive rather than proactive. ${ }^{26,51}$ While current maternal asthma guidelines recommend a written asthma action plan, longitudinal and prospective studies showed only $10-20 \%$ of females had a written asthma action plan throughout their pregnancy. ${ }^{26,35,51}$ These findings indicate that the majority of pregnant females self-manage their asthma, which may be problematic if they are unaware of how to appropriately respond to the changes in their asthma pathology. ${ }^{51}$

Another barrier limiting effective maternal asthma control is nonadherent medication use. For instance, a major reason for medication discontinuation relates to the perceived fear or lack of need to use asthma medications, particularly ICS. ${ }^{50}$ Fear-related suboptimal use during pregnancy has been reported in multiple countries, reflecting the global scale of this problem. ${ }^{35,52-55}$ Individuals were found to be unaware that their asthma symptoms could worsen during the course of their pregnancy, or considered medication use to be a greater concern than the potential risks associated with uncontrolled maternal asthma. ${ }^{35,52-55}$ Other reasons also included inadequate support and guidance from health professionals and their desire to seek alternative therapies. ${ }^{56}$

Physician reluctance to prescribe and lack of confidence to endorse asthma medication use is another barrier towards effective maternal asthma control. ${ }^{26}$ In a cross-sectional survey by Lim et al., ${ }^{57}>25 \%$ of pregnant patients with asthma were instructed by their family physician to cease and reduce asthma medication use despite having well-controlled asthma, while a French study by Beau et al. ${ }^{58}$ showed guidelines were not followed by all physicians. In particular, one-half of pregnant patients with asthma were not prescribed asthma medication on a regular basis and medications prescribed differed to those used during preconception, which does not reflect guideline recommendations to continue the same preconception asthma medication/s during pregnancy. ${ }^{58}$

Clearly, based on the evidence, it can be inferred that asthma management largely differs depending on the healthcare specialist and views of the patient, highlighting the discrepancy between clinical practice and current maternal asthma guidelines. This emphasises the importance of asthma education for the patients and multidisciplinary teams managing their asthma. It also reinforces the idea that multifaceted approaches should work synergistically for optimal management of asthma during pregnancy. ${ }^{50}$ A more current and pressing issue for pregnant individuals with asthma is the current outbreak of coronavirus disease (COVID-19) infection across the world and how it can be treated and managed in this vulnerable population.

\section{MATERNAL ASTHMA MANAGEMENT AND COVID-19}

In December 2019, an outbreak of pneumonia with unknown origins was identified in Wuhan, China. This was found to be caused by a novel coronavirus, ${ }^{59}$ now named severe acute respiratory syndrome coronavirus 2 (SARSCoV-2) by the World Health Organization (WHO) ${ }^{60}$ This virus causes COVID-19, a disease characterised by a severe respiratory illness similar to severe acute respiratory syndrome (SARS). ${ }^{61}$ The impact of the virus on pregnant females with asthma and their offspring is currently unknown, but we do know that the pregnant population has been at high risk of adverse outcomes in previous epidemics, with significantly higher rates of death during the 1918-1919 Spanish flu pandemic, 1957-1958 Asian flu pandemic, ${ }^{36} 2002$ SARS epidemic, and 2012 Middle East respiratory syndrome (MERS) epidemic. Case fatality rates for SARS and MERS were as high as $15 \%$ and $27 \%$, respectively, for pregnant females, although this is based on only a small number of reported patients with disease. ${ }^{37}$ 
To date, preliminary reports from China indicated that pregnancy does not aggravate the course of symptoms of COVID-1938 and, unlike SARS and MERS, there have been few maternal deaths associated with COVID-19 infection in pregnancy. ${ }^{62}$ A systematic review of pregnancy outcomes from COVID-19 indicated that preterm birth and perinatal death were the most common outcomes in pregnancies complicated by the virus, with limited evidence of vertical transmission of the virus to the fetus. ${ }^{63}$ In the USA, 15\% of COVID-19 infections in pregnancy were severe and occurred in females who had comorbidities that included obesity and asthma. ${ }^{64}$ Spanish data identified that $12.5 \%$ of pregnant patients with COVID-19 pneumonia have asthma. ${ }^{65}$ One maternal death reported in the UK occurred in a 29-year-old Pakistani female who had multiple comorbidities, including asthma. ${ }^{66}$ Brazilian data reporting COVID-19 maternal deaths associated with a comorbidity identified that $45 \%$ of deaths were pregnant females with asthma. ${ }^{67}$ Personalised predictive modelling for mortality, ventilation, and need for intensive care unit admission developed from Mexican data reported that pregnancy and asthma were predictors of these outcomes. ${ }^{68}$ These data suggest that severe COVID-19 infections arise in pregnant females with multiple comorbidities, including asthma, and that these females are at greater risk of intensive care unit admission and death. Furthermore, a prospective study examining viral infections in pregnant patients reported that those with asthma had a higher susceptibility to infection and more severe infections than those without asthma during pregnancy, ${ }^{69}$ which suggests pregnant females with asthma are an at-risk population for COVID-19 infection. Centers for Disease Control and Protection (CDC) guidelines indicate people with asthma may be at greater risk of more severe disease if infected with COVID-19, and there are data emerging that supports this information.

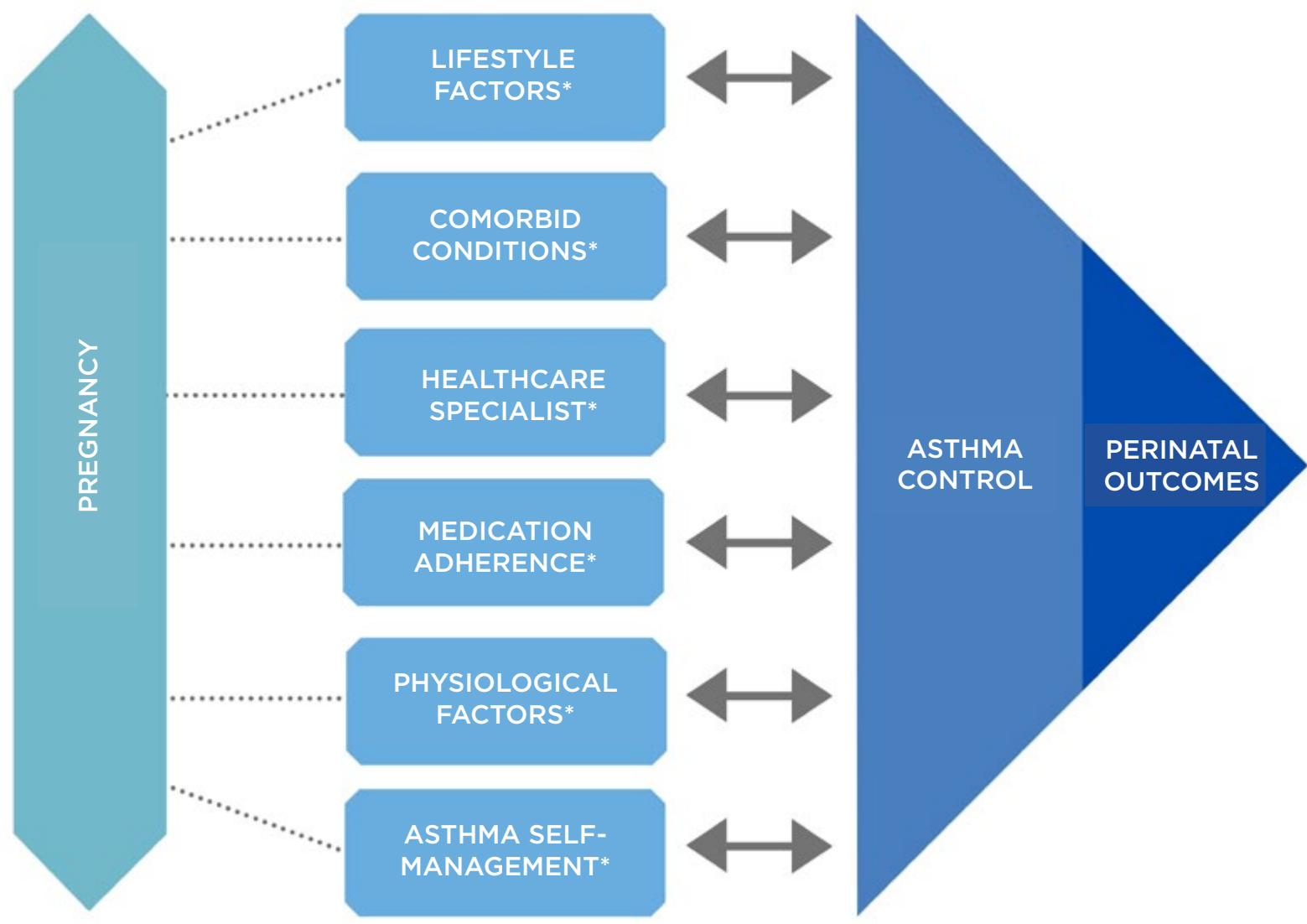

Figure 1: Variables that influence asthma control during pregnancy.

*Key interdependent factors known to affect asthma control and perinatal outcomes: factors may also influence each other. 
Treatment recommendations for people with asthma and COVID-19 infection remain the same as noninfected people with asthma, but to avoid nebuliser treatments due to aerosolisation of the virus and potential risk of transmission to others. ${ }^{70}$

\section{CONCLUSION}

Maternal asthma is a substantial burden for both the mother and the health system. With the current paucity of the ability of evidencebased strategies to improve adverse outcomes, it is important to understand that uncontrolled maternal asthma and subsequent exacerbations in antenatal settings can contribute to increased risks of adverse pregnancy outcomes (Figure 1). More research evaluating the relationship between COVID-19 and its effect on the state of asthma control during pregnancy should be considered to determine if the development of treatment strategies is required. Ultimately, there is a need for evidence-based clinical practice guidelines that translate current research findings to clinical practice and optimise asthma control within antenatal settings to potentially improve health outcomes for the mother and baby.

\section{References}

1. Australian Institute of Health and Welfare (AlHW). Asthma. 2020. Avilable at: https://www.aihw.gov. au/reports/chronic-respiratoryconditions/asthma/contents/asthma. Last accessed: 6 October 2020.

2. Grzeskowiak LE et al. Patterns, predictors and outcomes of asthma control and exacerbations during pregnancy: a prospective cohort study. ERJ Open Res. 2016;2(1):00054-2015.

3. Tara FC, Eugene B. Asthma heterogeneity and severity. World Allergy Organ J. 2016;9.

4. Zairina $\mathrm{E}$ et al. A prospective cohort study of pulmonary function during pregnancy in women with and without asthma. J Asthma. 2016;53(2):155-63.

5. Tamási L et al. Asthma in pregnancy - immunological changes and clinical management. Respir Med. 2011;105(2):159-64.

6. Wang $\mathrm{H}$ et al. Asthma in pregnancy pathophysiology, diagnosis, whole-course management, and medication safety. Can Respir J. 2020;2020:9046842.

7. Gold DR, Litonjua AA. Long-term benefits of optimal asthma control in pregnancy. J Allergy Clin Immunol. 2018;141(3):882-3.e1.

8. Murphy VE et al. Influence of maternal body mass index and macrophage activation on asthma exacerbations in pregnancy. J Allergy Clin Immunol Pract. 2017;5(4):981-7.e1.

9. Murphy VE et al. A meta-analysis of adverse perinatal outcomes in women with asthma. BJOG. 2011;118(11):1314-23.

10. Grzeskowiak LE et al. Strategies towards improving pharmacological management of asthma during pregnancy. Pharmacol Res. 2018;130:85-92.
11. British Thoracic Society; Scottish Intercollegiate Guidelines Network. British guideline on the management of asthma. Thorax. 2014;69 (Suppl 1):1-192.

12. Abdullah $\mathrm{K}$ et al. Effect of asthma exacerbation during pregnancy in women with asthma: a populationbased cohort study. Eur Respir J. 2020;55(2):1901335.

13. Tegethoff $M$ et al. Asthma during pregnancy and clinical outcomes in offspring: a national cohort study. Pediatrics. 2013;132(3):483-91.

14. Martel M-J et al. Maternal asthma, its control and severity in pregnancy, and the incidence of atopic dermatitis and allergic rhinitis in the offspring. $J$ Pediatr. 2009;155(5):707-13.e1.

15. Mitselou $\mathrm{N}$ et al. Adverse pregnancy outcomes and risk of later allergic rhinitis - nationwide Swedish cohort study. Pediatr Allergy Immunol. 2020;DOI:10.1111/pai.13230.

16. Global Initiative for Asthma (GINA). Global Strategy for Asthma Management and Prevention. 2020. Available at: https://ginasthma.org/. Last accessed: 4 Septermber 2020.

17. Asthma and pregnancy [published 2015]. In: eTG complete [digital]. North Melbourne: Therapeutic Guidelines Ltd.; 2020.

18. Clifton VL et al. Maternal and neonatal outcomes of pregnancies complicated by asthma in an Australian population. Aust N Z J Obstet Gynaecol. 2009;49(6):619-26.

19. Hodyl NA et al. Perinatal outcomes following maternal asthma and cigarette smoking during pregnancy. Eur Respir J. 2014;43(3):704-16.

20. Murphy VE et al. The effect of cigarette smoking on asthma control during exacerbations in pregnant women. Thorax. 2010;65(8):739-44.
21. Dombrowski MP. Asthma and pregnancy. Obstet Gynecol. 2006;108(3 Pt 1):667-81.

22. Schatz $M$ et al. The course of asthma during pregnancy, post partum, and with successive pregnancies: a prospective analysis. J Allergy Clin Immunol. 1988;81(3):509-17.

23. McLaughlin $\mathrm{K}$ et al. Review and appraisal of guidelines for the management of asthma during pregnancy. Women Birth. 2018;31(6):e349-57.

24. Robijn AL et al. Recent developments in asthma in pregnancy. Curr Opin Pulm Med. 2019;25(1):11-7.

25. Grzeskowiak L et al. An observational study of the impact of an antenatal asthma management service on asthma control during pregnancy. Eur J Obstet Gynecol Reprod Biol. 2016;197:48-53.

26. Grieger JA et al. Asthma control in pregnancy is associated with preconception dietary patterns. Public Health Nutr. 2016;19(2):332-8.

27. Powell $\mathrm{H}$ et al. Rhinitis in pregnant women with asthma is associated with poorer asthma control and quality of life. J Asthma. 2015;52(10):1023-30

28. Grzeskowiak LE et al. Impact of a history of maternal depression and anxiety on asthma control during pregnancy. J Asthma. 2017;54 (7):706-13

29. Powell $\mathrm{H}$ et al. Psychosocial outcomes are related to asthma control and quality of life in pregnant women with asthma. J Asthma. 2011;48(10):1032-40.

30. Powell $\mathrm{H}$ et al. Psychosocial variables are related to future exacerbation risk and perinatal outcomes in pregnant women with asthma. J Asthma. 2013;50(4):383-9. 
31. Tay TR, Hew M. Comorbid "treatable traits" in difficult asthma: current evidence and clinical evaluation. Allergy. 2018;73(7):1369-82.

32. Bidad K et al. Gastroesophagial reflux disease and asthma in pregnant women with dyspnea. Iran J Allergy Asthma Immunol. 2014;13(2):104-9.

33. Namazy JA, Schatz M Pharmacological difficulties in the treatment of asthma in pregnant women. Expert Rev Clin Pharmacol. 2017;10(3):285-92.

34. Grosso A et al. The course of asthma during pregnancy in a recent, multicase-control study on respiratory health. Allergy Asthma Clin Immunol. 2018;14(1):1-5.

35. Robijn AL et al. Trends in asthma self-management skills and inhaled corticosteroid use during pregnancy and postpartum from 2004 to 2017. J Asthma. 2019:56(6):594-602.

36. Schwartz DA, Graham AL. Potential maternal and infant outcomes from (Wuhan) coronavirus 2019-nCoV infecting pregnant women: lessons from SARS, MERS, and other human coronavirus infections. Viruses. 2020;12(2):194.

37. Mullins E et al. Coronavirus in pregnancy and delivery: rapid review. Ultrasound Obstet Gynecol. 2020;55(5):586-92

38. Liu D et al. Pregnancy and perinatal outcomes of women with coronavirus disease (COVID-19) pneumonia: a preliminary analysis. AJR Am Roentgenol. 2020;215(1):127-32.

39. Lim A et al. Systematic review of the safety of regular preventive asthma medications during pregnancy. Ann Pharmacother. 2011;45(7-8):931-45.

40. Murphy VE et al. The Breathing for Life Trial: a randomised controlled trial of fractional exhaled nitric oxide (FeNO)-based management of asthma during pregnancy and its impact on perinatal outcomes and infant and childhood respiratory health. BMC Pregnancy Childbirth. 2016;16(1):111.

41. Raherison $\mathrm{C}$ et al. [Woman's asthma throughout life: Towards a personalized management?]. Rev Mal Respir. 2020;37(2):144-60. (In French)

42. McLaughlin $\mathrm{K}$ et al. Fractiona exhaled nitric oxide-based asthma management: the feasibility of its implementation into antenatal care in New South Wales, Australia. Aust N Z J Obstet Gynaecol. 2020;60(3):389-95

43. Grzeskowiak LE, Clifton VL. Asthma management during pregnancy: how long before we can all breathe a little easier? J Asthma. 2015;52(10):1020-2.
44. Powell $\mathrm{H}$ et al. Management of asthma in pregnancy guided by measurement of fraction of exhaled nitric oxide: a double-blind randomised controlled trial. Lancet. 2011;378(9795):983-90.

45. Morten $\mathrm{M}$ et al. FeNO-guided management of asthma during pregnancy reduces respiratory symptoms and asthma diagnosis in childhood. Eur Resp J. 2016;48(s60).

46. Morten $M$ et al. Managing Asthma in Pregnancy (MAP) trial: FENO levels and childhood asthma. J Allergy Clin Immunol. 2018;142(6):1765-72.e4.

47. Williamson GR et al. Women's experiences of personalised support for asthma care during pregnancy: a systematic review of the literature. BMC Pregnancy Childbirth. 2017;17(1):69

48. Lim AS et al. Multidisciplinary approach to management of maternal asthma (MAMMA). Chest. 2014;145(5):1046-54.

49. Zairina E et al. Telehealth to improve asthma control in pregnancy: a randomized controlled trial. Respirology. 2016;21(5):867-74

50. Bain $\mathrm{E}$ et al. Interventions for managing asthma in pregnancy. Cochrane Database Syst Rev. 2014;2014(10):CD010660.

51. Ibrahim WH et al. Asthma knowledge, care, and outcome during pregnancy: the QAKCOP study. Chron Respir Dis. 2018;16:1479972318767719.

52. Murphy VE. Managing asthma in pregnancy. Breathe (Sheff). 2015;11(4):258-67.

53. Koo S-M et al. Effect of pregnancy on quantitative medication use and relation to exacerbations in asthma. BioMed Res Int. 2017;2017:8276190.

54. Kim S et al. Effect of pregnancy in asthma on health care use and perinatal outcomes. J Allergy Clin Immunol. 2015;136(5):1215-23.e6

55. Cleary BJ et al. Medication use in early pregnancy-prevalence and determinants of use in a prospective cohort of women. Pharmacoepidemiol Drug Saf. 2010;19(4):408-17.

56. Lim AS et al. Asthma during pregnancy: the experiences, concerns and views of pregnant women with asthma. J Asthma. 2012;49(5):474-9

57. Lim A et al. Management of asthma in pregnant women by general practitioners: a cross sectional survey. BMC Fam Pract. 2011;12:121.

58. Beau A-B et al. Prescription of asthma medications before and during pregnancy in France: An observational drug study using the EFEMERIS database. J Asthma. 2017;54(3):258-64.
59. Zhu $\mathrm{N}$ et al. A novel coronavirus from patients with pneumonia in China, 2019 N Engl J Med. 2020;382(8):727-33.

60. World Health Organization (WHO). Naming the coronavirus disease (COVID-19) and the virus that causes it. 2020. Available at: https://www. who.int/emergencies/diseases/ novel-coronavirus-2019/technicalguidance/naming-the-coronavirusdisease-(covid-2019)-and-the-virusthat-causes-it. Last accessed: 4 September 2020.

61. Huang $\mathrm{C}$ et al. Clinical features of patients infected with 2019 novel coronavirus in Wuhan, China. Lancet. 2020;395(10223):497-506.

62. Schwartz DA. An analysis of 38 pregnant women with COVID-19, their newborn infants, and maternalfetal transmission of SARS-CoV-2: maternal coronavirus infections and pregnancy outcomes. Arch Pathol Lab Med. 2020;DOI:10.5858/ arpa.2020-0901-SA. [Epub ahead of print]

63. Di Mascio D et al. Outcome of coronavirus spectrum infections (SARS, MERS, COVID 1 -19) during pregnancy: a systematic review and meta-analysis. Am J Obstet Gyneco MFM. 2020:100107.

64. Lokken EM et al. Clinica characteristics of 46 pregnant women with a SARS-CoV-2 infection in Washington State. Am J Obstet Gynecol. 2020;DOI:10.1016/j. ajog.2020.05.031. [Epub ahead of print].

65. San-Juan R et al. Incidence and clinical profiles of COVID-19 pneumonia in pregnant women: a single-centre cohort study from Spain. EClinicalMedicine. 2020;23:100407.

66. Ahmed I et al. First COVID-19 maternal mortality in the UK associated with thrombotic complications. Br J Haematol. 2020;190(1):e37-8.

67. Takemoto MLS et al. Materna mortality and COVID-19. J Matern Fetal Neonatal Med. 2020:1-7. [Epub ahead of print]

68. Wollenstein-Betech S et al. Personalized predictive models for symptomatic COVID-19 patients using basic preconditions: hospitalizations, mortality, and the need for an ICU or ventilator. medRxiv. 2020; 2020;05.03.20089813. [Preprint]

69. Murphy VE et al. A prospective study of respiratory viral infection in pregnant women with and without asthma. Chest. 2013;144(2):420-7.

70. Pennington $\mathrm{E}$. Asthma increases risk of severity of COVID-19. Cleve Clin J Med. 2020;DOI:10.3949/ ccjm.87a.ccc002. 